\title{
A noise calculation and experiment with analog ROF transmission module
}

\author{
Xiaojing Wang ${ }^{1,}$, Mingyu Lin ${ }^{1}$, Yaoting Yang ${ }^{2}$ and Guihua Han $^{1}$ \\ ${ }^{1}$ Mechanical and Information Engineering College, Hubei Business College \\ ${ }^{2}$ Wuhan Hongxin Telecommunications Technologies Co., Ltd
}

\begin{abstract}
ROF technology is widely applied in high speed wireless communications of large capacity, ROF system can introduce noise simultaneously, affects the system's noise figure. This paper calculated in detail the noise figure of the analog ROF transmission module, used the classic noise theory of the analog optical fiber communication system, verified them combining with laboratory tests. Finally this paper analyzed some problems in its engineering application. It is the first time to provide a method for noise figure calculation in the ROF system engineering application.
\end{abstract}

Keywords: carrier-to-noise ratio; analog ROF transmission module; noises figure.

The analog ROF transmission module is widely used in all kinds of the interior and outdoor transmission and coverage system such as the relay transmission and optical distribution system of CATV and mobile communication network (including a variety of formats and frequency bands about 2G, 3G and 4G) and so on. ROF system introduces noise and affects the system's noise figure, while analog ROF transmission module has a crucial influence on the system equipments (especially uplink), then will affect the receiving sensitivity of the receiver [2]. But about the specific calculation of the ROF system noise, we have not seen some related literatures discussed. In this paper, we calculated in detail the noise figure of the analog ROF transmission module, and verified them combining with laboratory tests, discussed the related problems, at last we analyzed some problems in its engineering application, provided a noise figure calculation method in the ROF system engineering application.

\section{Carrier-to-noise ratio of analog ROF transmission system}

In analog ROF transmission system, when an optical detector has no gain, the main sources of noises are including the optical intensity noise of the laser maser, the quantization noise, thermal noise and noise generated by dark current of the detector. In the semiconductor laser, the amplitude fluctuation of the output optical produces the optical intensity noise[2]; The quantization noise is caused by photoelectron, when optical signals incident into the optical detector, the generation and collection of the photoelectron obeys the Poisson distribution with statistical properties, the number of the photon-generated carrier caused by the photoelectric effect is random fluctuation; The thermal noise refers to the thermal noise of the detector load resistor, whose cause is random thermal motion of the electrons; The dark current noise is the noise produced by the bias circuit current of the optical

a Corresponding author : jsgn0923@163.com

(C) 2016. The authors - Published by Atlantis Press 
detector when there is no light incident, including the body dark current noise and the surface dark current (surface leakage current) noise. In addition, the luminous power received by the detector and the laser modulation mode determine the carrier-to-noise ratio of the analog ROF transmission system. Assumes that the laser uses direct amplitude modulation, the expression of the carrier-to-noise ratio is as follows[1,6,7]:

$$
\frac{C}{N}=\frac{\frac{1}{2}(m \gamma M P)^{2}}{R I N(\gamma P)^{2} B+2 q\left(I_{P}+I_{D}\right) M^{2} F(M) B+2 q I_{L} B+4 k_{B} T B / R_{L}}
$$

Among them, $R I N(\gamma P)^{2} B$ is the laser's optical intensity noise; $2 q I_{P} M^{2} F(M) B$ is the detector's quantization noise; $4 k_{B} T B / R_{L}$ is the detector's thermal noise; $2 q I_{D} M^{2} F(M) B$ is the detector's body dark current noise; $2 q I_{L} B$ is the detector's surface dark current noise.

Here, $\mathrm{m}$ is signal modulation depth; $\gamma$ is the detector's responsivity; $M$ is the optical detector's gain; $p$ is the average accepted luminous power; $R I N$ is the laser relative noise intensity; $B$ is the equivalent noise bandwidth; $q$ is electronic charge; $I_{p}$ is primary photocurrent, with $\gamma{ }^{*} p$; $I_{D}$ is the primary body current; $F(M)$ for the photodiode's excess noise figure, with $M^{x}(0 \leq x \leq 1) ; \mathrm{K}_{\mathrm{B}}$ is the Boltzmann constant; $T$ is absolute temperature; $I_{L}$ is the surface leakage current; $R_{L}$ is the optical detector load resistance.

When the accepted luminous power is very high, the laser's optical intensity noise becomes the main noise; while for the medium sized accepted luminous power, the main noise is the quantization noise; when it is low, the thermal noise of the receiver becomes the main noise[3,5].

This paper applied the classic noise theory of the analog optical fiber communication system above to the ROF system's noise calculation.

\section{Noise figure of analog ROF transmission module}

The RF link block diagram of the ROF transmission module we adopted is as follows[8]:

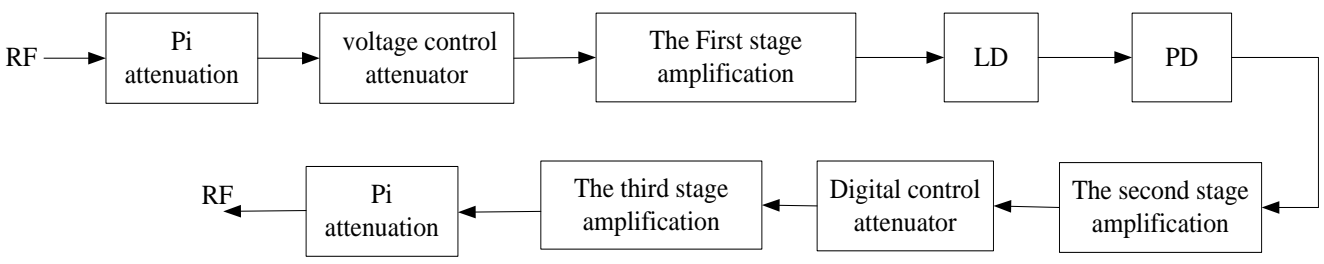

Figure 1. ROF transmission module link model in Laboratory

From the above link block diagram, the noises of the ROF transmission module were decided together by the noises of the amplifier and the attenuator of the each RF devices and the laser-detector circuit. The noise of the laser-detector circuit was particularly important. Through the calculation of the noise of this part circuit, then the noise figure of module was calculated.

The laser model we chose was RLD - D3S1A, whose parameters are as follows:

Threshold current $I_{t h}$ : $10.5 \mathrm{~mA}$; Working current $I_{b}$ : $26 \mathrm{~mA}$;

The average output optical power $P: 2.45 \mathrm{~mW}$; Center wavelength $\lambda: 1310 \mathrm{~nm}$;

Relative intensity noise $R I N$ : $-155 d B c$;

The detector model was RPDS- P062AH, its parameters are as follows:

responsivity $\gamma$ : 0.98; The body dark current $I_{D}=1 n A$;

By the carrier-to-noise ratio formula of the analog ROF transmission, the modulation depth $\mathrm{m}$ has a direct impact on the carrier-to-noise ratio. In practical application, in order not to give the optical signal introducing into distortion, modulation must limit in the linear part of the optical source output 
curve, $m$ value ranges from 0.25 to 0.5 . In our RF optical module, RF power of the input laser was $0 \mathrm{dBm}$, the corresponding current was $4.48 \mathrm{~mA}$, the modulation m was 0.29 ; Module adopted PIN tube to receive, thus the $M$ and $F(M)$ values are both equal to 1 . Noise bandwidth $B$ was $1 \mathrm{~Hz}$; the noise caused by the dark current is very small, ignore this part noise in calculation; Detector load $R_{L}$ was $1 k \Omega$; Boltzmann constant $k_{B}=1.38 \times 10^{-23} \mathrm{~J} / \mathrm{K}$; Absolute temperature $T=293 \mathrm{~K}$; We put the above values into the carrier-to-noise ratio formula of the analog ROF transmission, and assuming that the optical link without attenuation, thus the luminous power was equal to the accepted power, get the carrier-to-noise ratio in this moment was: $C_{0} / N_{0}=140 \mathrm{~dB}$

The signal input SNR of the input laser-detector circuit was: $C_{i} / N_{i}=174 d B$

The noise figure of the laser-detector circuit:

$$
N F=\left(C_{i} / N_{i}\right) /\left(C_{0} / N_{0}\right)=34 d B
$$

The distribution of the gain and noise figure of each part of the ROF module we designed was as follows

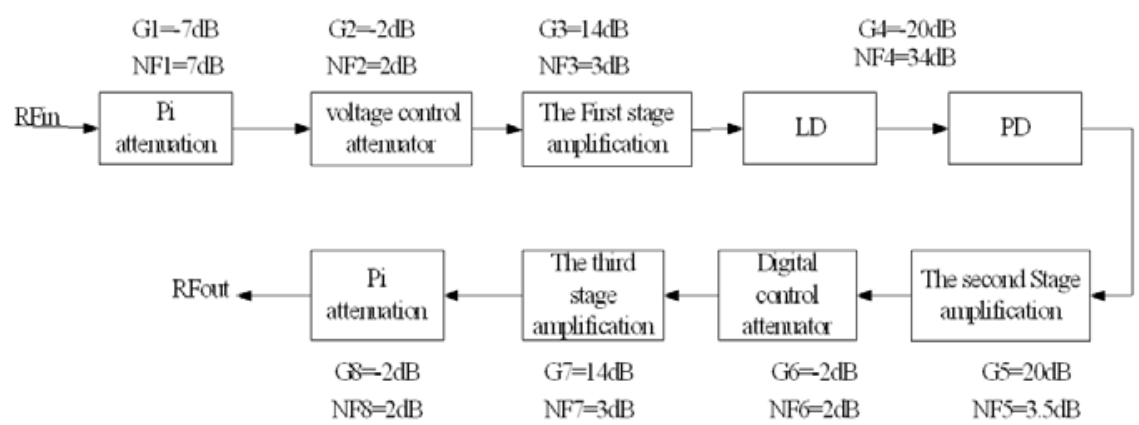

Figure 2. ROF transmission module gain and noise system distribution

According to the noise figure formula of the cascade system, the total noise figure of the ROF transmission module was as follows:

$$
\begin{gathered}
N F_{\text {total }}=N F 1+\frac{N F 2-1}{G 1}+\frac{N F 3-1}{G 1 G 2} \ldots \ldots \ldots \ldots \ldots . . \frac{N F 8-1}{G 1 G 2 G 3 G 4 G 5 G 6 G 7} \\
N F_{\text {total }} \approx 851.1=29.3 \mathrm{~dB}
\end{gathered}
$$

\section{Noise experiment of analog ROF transmission module}

In the experiments, we input 900M RF signal whose input range was $-5 \mathrm{dBm}$ in the RF optical module input end, the laser luminous power was $4 \mathrm{dBm}$, the optical link attenuation was $0 \mathrm{~dB}$, the RF link gain was $22 \mathrm{~dB}$ (- $40 \mathrm{dBm}$ signal input, $17.8 \mathrm{dBm}$ output), the optical module RF output end bottom noise was $-123 \mathrm{dBm} / \mathrm{Hz}$ measured by spectrometer, thus the noise figure of ROF transmission module obtained was: $N F_{\text {total }}=29 d B$.

Consistent with the calculation results, 900M signal output power and bottom noise experiment screenshots were as follows: 


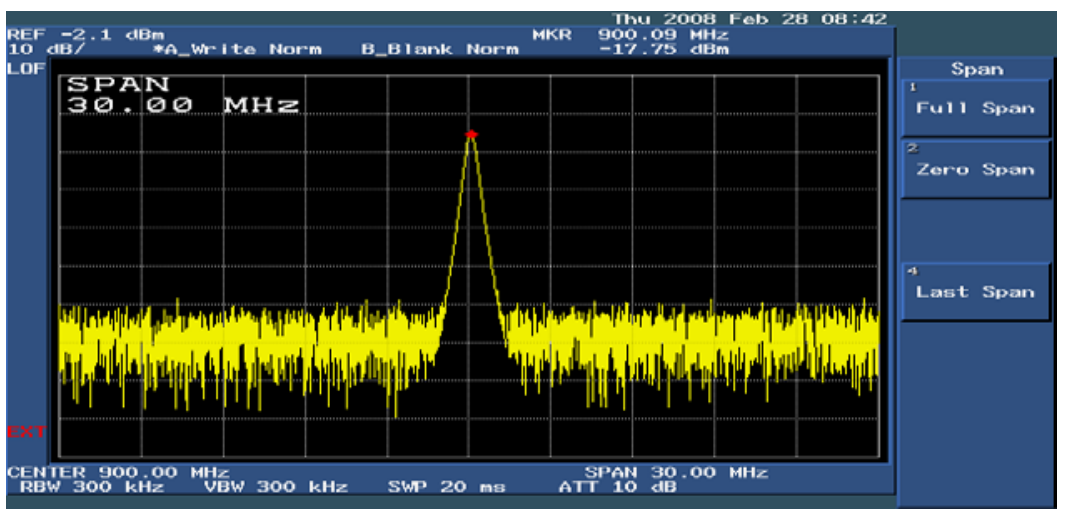

Figure 3. ROF transmission module output signal power

In figure 3 , the power output of the visible module is $-17.75 \mathrm{dBm}$, and the output signal frequency is $900 \mathrm{MHZ}$.

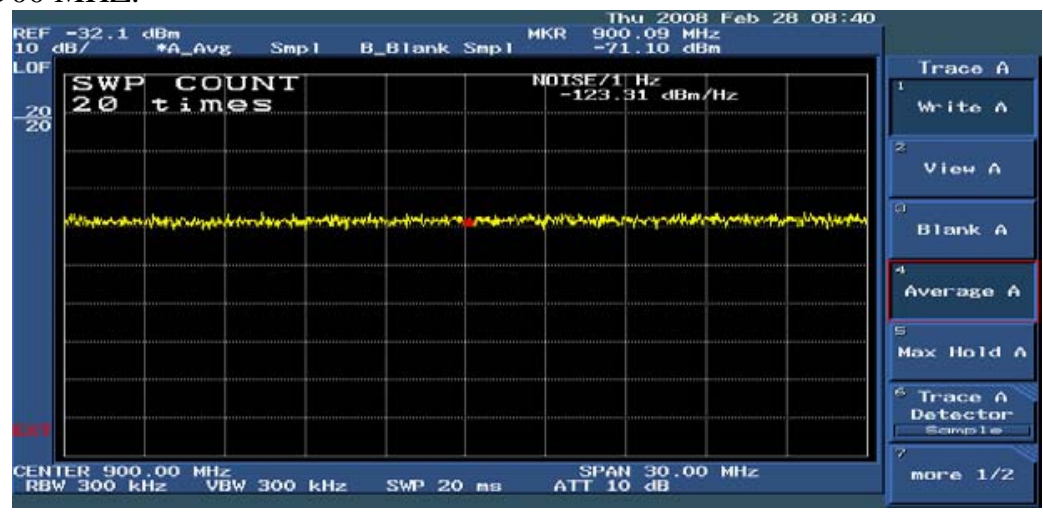

Figure 4. ROF transmission module bottom noise

In figure 4 , the noise power of the display module bottom is $-123 \mathrm{dBm} / \mathrm{Hz}$. While white noise power is $-174 \mathrm{dBm} / \mathrm{Hz}$, the module gain is $22 \mathrm{~dB}$, the module noise figure is $29 \mathrm{~dB}$, so the module bottom noise is: $-174+22+29=-123(\mathrm{dBm} / \mathrm{Hz})$.

\section{Analysis problems in engineering application for analog ROF transmission module}

In engineering applications, in order to satisfy the system gain requirement, it needs to attenuate the ROF transmission module gain, the gain attenuation is divided into these two ways between circuit and optical path attenuation. But the noise figure changes of the ROF transmission module by these two methods caused are not the same. Now based on the circuit structure of the ROF transmission module we designed, assuming that RF gain attenuation was between $0 \mathrm{~dB}$ and $40 \mathrm{~dB}$, we would discuss the noise figure change of the ROF transmission module by these two methods.

\subsection{Circuit attenuation}

In the cause of the circuit attenuation, the noise and loss of the laser-detector didn't change, while the entire link noise increased. We put the attenuation values between $0 \mathrm{~dB}$ and $40 \mathrm{~dB}$ into (3), the result is shown in figure 5 that the noise figure of the ROF transmission module simulated by the software which was along with the attenuation change. When the circuit attenuation was $20 \mathrm{~dB}$ and below, the which was along with the attenuation change. When the circuit attenuation was $20 \mathrm{~dB}$ and below, the 
noise figure change of the ROF module was smaller; the noise system was deteriorated significantly when the circuit attenuation was increased more than $20 \mathrm{~dB}$. But the $40 \mathrm{~dB}$ noise figure was still within the acceptable range of the system budget.

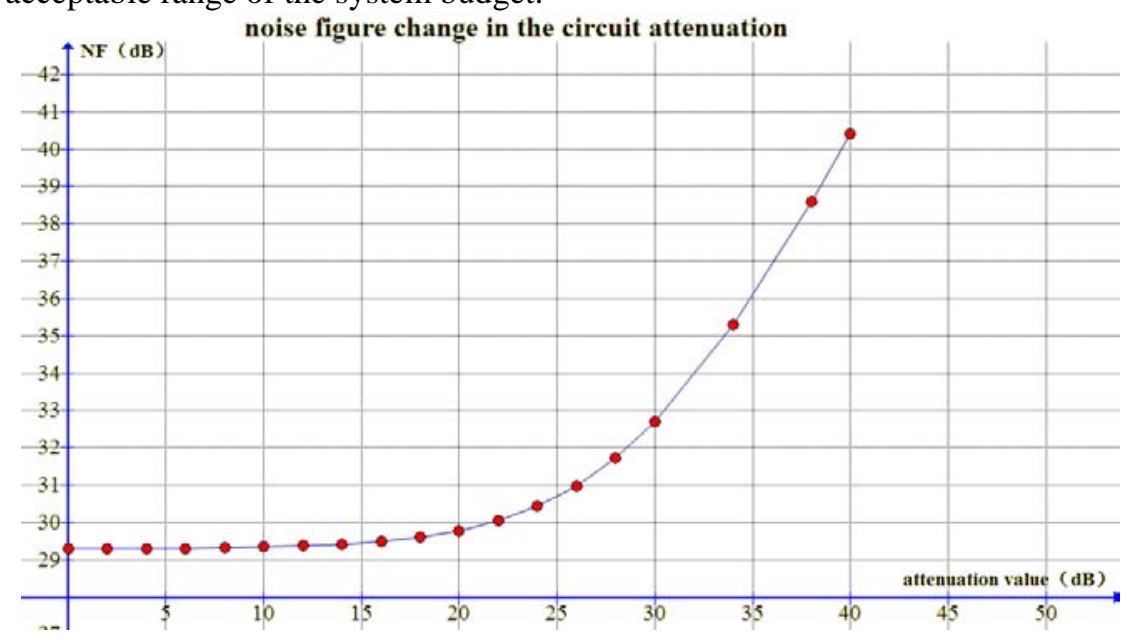

Figure 5. ROF transmission module noise figure in circuit attenuation

\subsection{Optical path attenuation}

In the cause of the optical path attenuation, the laser's luminous power and SNR were constant, the accepted optical power was declined, the whole link noise was violently increased. We put the optical attenuation values between $0 \mathrm{~dB}$ and $20 \mathrm{~dB}$ (The relationship between the optical path attenuation and circuit attenuation is multiple, namely the optical path attenuation value is the corresponding circuit attenuation value two times) into (1), calculated the noise figure of the laser-detector circuit, then put it into (3), calculated the noise figure of the ROF transmission module, get the noise figure along with the attenuation change was shown in figure 6 simulated by the software. In the process of the optical path attenuation, the noise system of the ROF transmission module was deteriorated significantly, and the noise system has deteriorated to nearly $40 \mathrm{~dB}$ when optical attenuation was $10 \mathrm{~dB}(20 \mathrm{~dB}$ corresponding to the circuit attenuation), the situation which continued to worsen would not be accepted by the budget system.

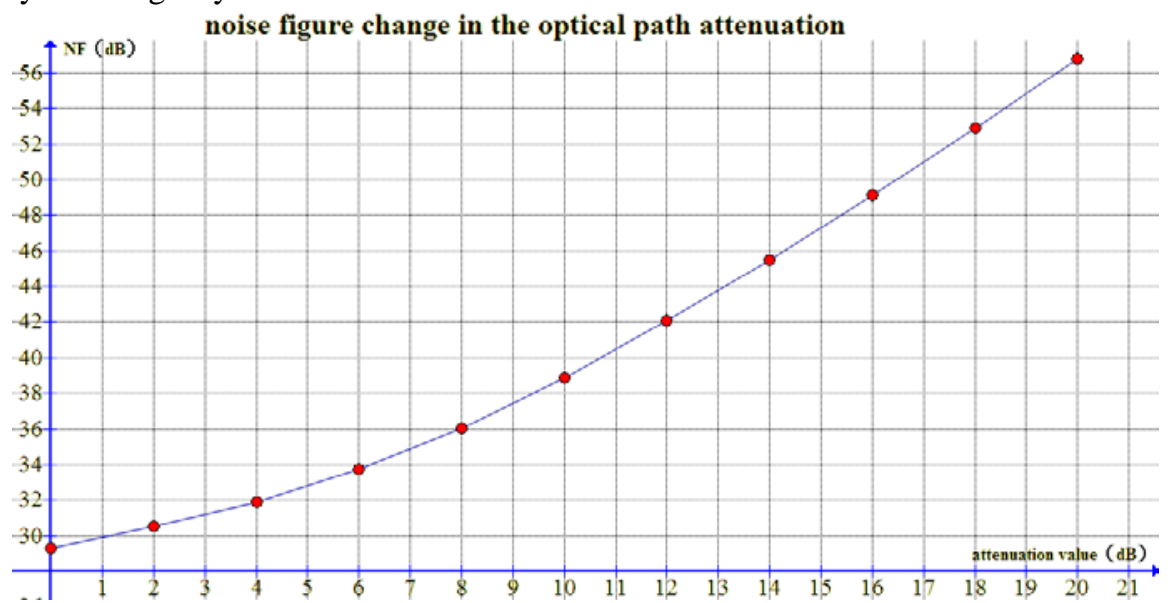

Figure 6. ROF transmission module noise figure in optical attenuation 


\subsection{Summary}

As known from the analysis above, optical path attention and circuit attenuation both make the noise figure of the ROF transmission module to increase, and provide a calculating noise figure method in different engineering application cases. Optical path attention makes the detector's SNR sharply felling, the noise figure of the laser- detector circuit is obvious rise, which causes the entire link noise increase. And noise increase caused by circuit attenuation has less impact than optical path attention. So in engineering application, in addition to the necessary optical path attenuation (such as fiber loss, light path for splitter and combiner, etc.), should try to reduce unnecessary optical path attenuation, to reduce the effect of system noise, if it is need to adjust the system gain, appropriate gain adjustment should adopt circuit attenuation of the module link.

\section{Conclusion}

This paper calculated in detail the noise figure of a ROF transmission module on the base of the carrier-to-noise ratio formula of the analog optical fiber transmission system, and verified it combining with laboratory tests, the problem about the noise system larger of the ROF transmission module was analyzed. At the same time, the link gain attenuation problem in engineering application was discussed in this paper, we pointed out the specific impacts of the noise figure caused by the circuit attenuation and optical attenuation, providing guidance for engineering practices.

\section{References}

1. Joseph.C.Palais, Optical Fiber Communication (the fifth edition) (published) Publishing House of Electronics Industry (2015)

2. Xavier. N. fernando, ROF Optical Wireless Communication: From Theory to Forefront (published) China Machine Press (2015)

3. DI Zhu, IY Denisyuk, MI Fokina, Optical radio-photonic channel for transmission of a coherent narrowband analog signal (Optics \& Spectroscopy, 2013)

4. Cen S.Z. Hu B. Zhu S.L .Wei B, Several Important Indexes and Test Methods in Analog Signals Optical Transceiver (Optical communication technology, 2013)

5. J. Capmany, .Mora, I. Gasulla, J.Sancho, J. Lloret, and S. Sales, Microwave photonic singal processing (Journal of Lightwave Technology, 2013)

6. ZuoL. Hou S.H, Analysis of Noise Figure in ROF Modulation Outside Link (Communications technology, 2012)

7. Zhu D.W. Zhu S.L, Xi H.B, Analysis of Noise Figure of Analog Signal Optical Fiber Transmission System (Optical communication technology, 2011)

8. D.Wake,A.Nkansah,andN.J.Gomes, Radio over fiber link design for next generation wireless systems(Journal of Lightwave Technology, 2010) 